\title{
New prospects for the rational design of antivirals
}

\author{
Estanislao Nistal-Villán \& Adolfo García-Sastre
}

Despite the availability of influenza vaccines, influenza virus continues to be a worldwide public health burden. During typical epidemic years, approximately 36,000 people die and 200,000 are hospitalized just in the US as a result of severe complications associated with influenza virus infections ${ }^{1}$. These numbers can dramatically increase in severe pandemic years. One of the most extreme examples of how damaging influenza can be was the 1918-1919 pandemic. This pandemic, caused by an H1N1 virus, resulted in 50 million deaths, mainly among the adult population, and had devastating consequences for humankind ${ }^{2}$.

The new $\mathrm{H} 1 \mathrm{~N} 1$ virus that is causing the first influenza virus pandemic of this century is clearly less virulent than the 1918 virus. But this pandemic episode has given us sobering lessons on our ability to combat pandemic influenza.

For instance, it has reminded us of our inability to predict the specific subtype that will start a new pandemic. Whereas most attention was given to $\mathrm{H} 5$ and, to some extent, $\mathrm{H} 7, \mathrm{H} 9$ and $\mathrm{H} 2$ viruses as potential new pandemic strains, $\mathrm{H} 1$ viruses were under the radar because there was no precedent of an influenza pandemic caused by an $\mathrm{H} 1$ subtype virus circulating in humans. However, the large antigenic differences between the new swine-origin $\mathrm{H} 1 \mathrm{~N} 1$ virus and the previously circulating human $\mathrm{H} 1 \mathrm{~N} 1$ viruses, responsible for the limited pre-existing immunity in humans against swine $\mathrm{H} 1 \mathrm{~N} 1$ viruses, most likely facilitated the species jump from a pig reservoir into humans ${ }^{3-5}$. Moreover, the extended influenza virus surveillance programs in humans allowed us to conclude that the new $\mathrm{H} 1 \mathrm{~N} 1$ virus started a pandemic with almost no preceding period of adaptation to humans, challenging our

Estanislao Nistal-Villán and Adolfo García-Sastre are at the Department of Microbiology, Mount Sinai School of Medicine, New York, New York, USA. Adolfo García-Sastre is also affiliated with the Department of Medicine, Division of Infectious Diseases and the Global Health and Emerging Pathogens Institute of Mount Sinai School of Medicine, New York, New York, USA. e-mail: adolfo.garcia-sastre@mssm.edu prospects of producing a vaccine against the new pandemic virus in time to protect the human population. Thus, although a vaccine is now available, no vaccination was possible during the winter season in the Southern Hemisphere, where the new $\mathrm{H} 1 \mathrm{~N} 1$ virus was prevalent.

During the lag time needed to produce vaccines, our only weapons against pandemic influenza viruses are antivirals. And, even during typical epidemic years, because vaccination does not completely prevent influenza virus infection, antivirals are also crucial for the therapeutic treatment of influenza.

Two classes of antivirals have been approved by the US Food and Drug Administration for the treatment of influenza: the M2 channel blockers amantadine and rimantadine and the neuraminidase inhibitors oseltamivir and zanamivir. The first class inhibits the ability of the viral M2 protein to pump protons, a function required for viral uncoating during the first steps of viral entry into the host cell. By contrast, the second class inhibits the enzymatic activity of the viral NA protein, needed for the spread of the influenza virions newly generated by the infected host cell.

A major concern, however, for the use of these antivirals is the widespread resistance of circulating influenza viruses to them. The majority of the human circulating $\mathrm{H} 3 \mathrm{~N} 2$ viruses are resistant to amantadine and rimantadine, whereas the human $\mathrm{H} 1 \mathrm{~N} 1$ viruses of the last season have acquired oseltamivir resistance ${ }^{6}$. The new $\mathrm{H} 1 \mathrm{~N} 1$ pandemic viruses are resistant to the $\mathrm{M} 2$ channel blockers but sensitive to neuraminidase inhibitors. However, oseltamivir-resistant isolates have already been described, and they may become prevalent under selection pressure. Clearly, new antivirals are needed to efficiently combat influenza viruses.

A potential new target for the development of influenza antiviral compounds is the viral RNA-dependent RNA polymerase. This viral enzyme, required for viral RNA replication and transcription, has several unique enzymatic properties that make it ideal for the development of specific antivirals. In fact, inhibitors of the influenza polymerase activity have already been described ${ }^{7}$, and they have

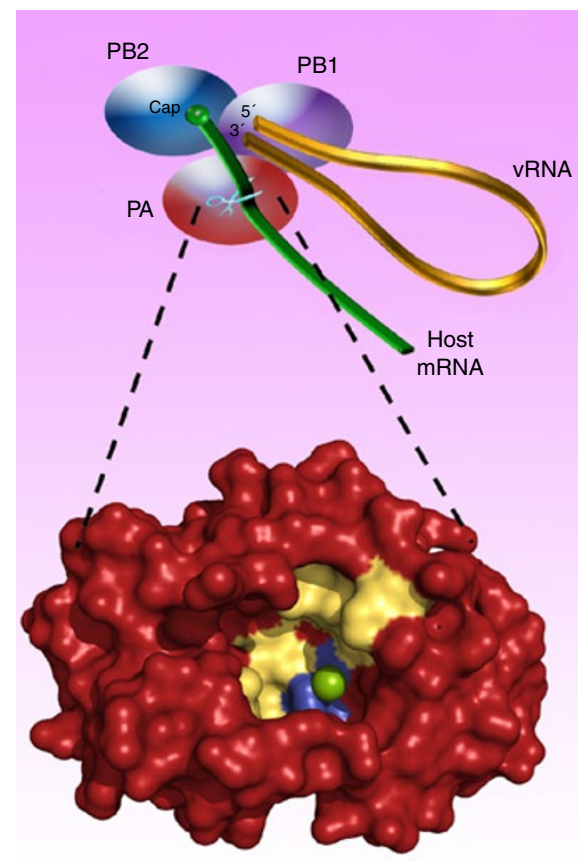

Figure 1 A new antiviral target. Schematic representation of the initiation of viral mRNA synthesis by the influenza virus RNA polymerase and of the structure of its endonuclease active site. The polymerase is comprised of three subunits, PB1, PB2 and PA. The PB1 subunit binds the $5^{\prime}$ and $3^{\prime}$ ends of the viral RNA template (in yellow), whereas the PB2 subunit binds the $5^{\prime}$ capped ends of a host cellular mRNA (in green). Cleavage of the cellular mRNA mediated by the endonuclease domain of the PA subunit generates the primers used by the RNAdependent RNA polymerase domain of the PB1 subunit for viral mRNA synthesis. The structure of the endonuclease active site is represented in the bottom of the figure (Protein Data Bank accession number 3EBJ), and it reveals a nucleoside monophosphate binding pocket (in yellow), where amino acid residues (in blue) binding to $\mathrm{Mg}^{2+}$ (in green) are also located ${ }^{13-15}$. This pocket represents a new target for the development of small-molecule inhibitors. Adapted from ref. 15.

the potential to become new antiviral drugs if proven safe and efficacious in humans. However, one of the hurdles in the rational design of influenza virus RNA polymerase inhibitors has been the lack of detailed structures of the polymerase's enzymatic domains required for RNA transcription. 
The influenza viral RNA polymerase is a heterotrimeric complex composed of three subunits, PB2, PB1 and PA (Fig. 1). During viral messenger RNA synthesis, the PB1 subunit binds the promoter of the influenza virus RNA genes. This promoter is formed by the very terminal sequences at the $3^{\prime}$ and $5^{\prime}$ ends of the virus RNA genes. By contrast, the PB2 subunit binds $5^{\prime}$ cap structures on cellular mRNAs. The cellular mRNAs bound to $\mathrm{PB} 2$ are subsequently cleaved, generating short, capped RNA oligonucleotides that are used as primers for the initiation and elongation of viral mRNA synthesis. Thus, two enzyme activities are associated with viral mRNA synthesis by the influenza virus polymerase: an endonuclease that cleaves the cellular mRNAs, a process also referred to as 'cap snatching', and an RNA-dependent RNA polymerase that elongates the capped primers using viral RNAs as templates. The PB1 subunit contains the RNA polymerase domain, and for a long time it was believed that the PB1 subunit was also responsible for the endonuclease activity, whereas the role of the PA subunit in the heterotrimeric complex was unclear.

Recently, the crystal structures of some domains of the viral polymerase associated with protein-protein or protein-RNA interactions have been generated. These include the contact domains between the PB1 and PA proteins $^{8,9}$ and between the PB1 and PB2 proteins ${ }^{10}$, the PB2 cap-binding domain ${ }^{11}$ and the nuclear localization signal of PB2 complexed with importin- $\alpha 5$ (ref. 12). Although these complexes represent potential targets for antiviral compounds meant to inhibit crucial interactions of the viral polymerase, the structure of the whole polymerase complex or of the enzymatic domains of the polymerase will help in the rational design of small molecules that target the active sites of this viral enzyme.

A recent breakthrough was achieved by Yuan et al. ${ }^{13}$ and Dias et al. ${ }^{14}$, who indepen- dently determined the crystal structures of the first 197 and 209 amino acid residues of the PA subunit, respectively.

Surprisingly, the structure revealed that this domain contains the endonuclease active site of the influenza virus polymerase complex, in contrast to the belief that this activity was located in the PB1 subunit (Fig. 1). The structure also allowed the identification of amino acid residues, conserved in all influenza viruses, that bind metal ions and form the endonuclease active site. Mutations at these amino acids abolished the endonuclease activity of the influenza viral polymerase complex.

This information now provides a structural framework for the identification of small molecule compounds that bind the endonuclease and inhibit this viral enzyme. Such inhibitors are predicted to prevent viral mRNA synthesis and therefore viral replication. Even more recently, Zhao et al. ${ }^{15}$, following on the preceding PA endonuclease structure, conducted a crystallographic analysis of this domain complexed with nucleoside monophosphates. These studies identified a distinct site inside the catalytic center of the enzyme that binds ribo-UMP (rUMP), rAMP and TMP but not rGMP or rCMP, highlighting a nucleotide binding pocket for potential inhibition by small molecule compounds.

The rapid pace by which the structures of domains of the influenza virus polymerase have been resolved also provides hope that the X-ray structure of the whole heterotrimeric complex of the viral RNA polymerase will be determined in the near future. Such a structure will help in the rational design of inhibitors against the various active domains of the viral polymerase.

The use of inhibitors against multiple domains of the influenza virus polymerase in combination with other antiviral therapies should lower the probability of the emergence of resistant strains of influenza viruses. Moreover, the identification of cellular factors required for influenza virus replication may also expand the number of potential targets for the development of antiviral compounds. Ongoing studies using proteomics and siRNA high-throughput screening techniques to determine the subset of cellular proteins and pathways that comprise the functional influenza virus interactome is likely, in the near future, to uncover cellular targets for influenza treatment.

Even if new classes of inhibitors that efficiently reduce viral replication are developed, the challenges associated with the short window of time for effective intervention against influenza may still remain. The antivirals already in use are only effective if individuals are treated early during infection, most likely because the appearance of disease symptoms precedes the peak of influenza viral replication by only a few days. Thus, any effective therapy for influenza virus requires the development of rapid diagnostic techniques in addition to effective and safe antiviral drugs.

In the absence of an effective universal vaccine against influenza virus, more reliable and faster diagnostic methods and new antivirals are required to reduce the burden imposed by epidemic and pandemic influenza.

1. Thompson, W.W. et al. J. Am. Med. Assoc. 289, 179186 (2003)

2. Johnson, N.P. \& Mueller, J. Bull. Hist. Med. 76, 105 115 (2002).

3. Garten, R.J. et al. Science 325, 197-201 (2009).

4. Itoh, Y. et al. Nature 460, 1021-1025 (2009).

5. Hancock, K. et al. N. Engl. J. Med. published online, doi:10.1056/NEJMoa0906453 (10 September 2009).

6. Moscona, A. N. Engl. J. Med. 360, 953-956 (2009).

7. Beigel, J. \& Bray, M. Antiviral Res. 78, 91-102 (2008).

8. Obayashi, E. et al. Nature 454, 1127-1131 (2008).

9. He, X. et al. Nature 454, 1123-1126 (2008).

10. Sugiyama, K. et al. EMBO J. 28, 1803-1811 (2009).

11. Guilligay, D. et al. Nat. Struct. Mol. Biol. 15, 500-506 (2008).

12. Tarendeau, F. et al. Nat. Struct. Mol. Biol. 14, 229-233 (2007).

13. Yuan, P. et al. Nature 458, 909-913 (2009).

14. Dias, A. et al. Nature 458, 914-918 (2009).

15. Zhao, C. et al. J. Virol. 83, 9024-9030 (2009). 